\title{
Noise Suppression via Atomic Absorption in a Raman Quantum Memory
}

\author{
T.M. Hird ${ }^{1,2}$, S.E. Thomas ${ }^{1,3}$, J.H.D. Munns ${ }^{1,3}$, B. Brecht ${ }^{1,4}$, D.J. Saunders ${ }^{1}$, \\ J. Nunn ${ }^{1,5}$, I.A. Walmsley ${ }^{1,3}$, P.M. Ledingham ${ }^{1}$ \\ ${ }^{1}$ Clarendon Laboratory, University of Oxford, Parks Road, Oxford, OX1 3PU, UK \\ ${ }^{2}$ QOLS, Department of Physics, Imperial College London, London SW7 2BW, UK \\ ${ }^{3}$ Department of Physics and Astronomy, University College London, London WCIE 6BT, UK \\ ${ }^{4}$ Applied Physics, University of Paderborn, Warburgerstrasse 100, 33098 Paderborn, Germany \\ ${ }^{5}$ Centre for Photonics and Photonic Materials, University of Bath, Bath, BA2 7AY, UK \\ thomas.hird@physics.ox.ac.uk
}

\begin{abstract}
We propose and demonstrate a scheme to strongly suppress four-wave mixing noise on the output of a Raman quantum optical memory. We show that heralded singlephoton states can be recalled nonclassically using our device. (C) 2018 The Author(s)
\end{abstract}

OCIS codes: $270.0270,270.4180,270.5585$

Single-mode broadband quantum memories provide unique capabilities that can enhance and expand the performance of future quantum networks. On the one hand, they facilitate the temporally multiplexed generation of pure single photons at high clock rates, which can dramatically increase photon generation rates [1]. On the other hand, their single mode nature allows for operation as a broadband beam splitter that can operate on novel bases for information encoding. Their usefullness within quantum architectures hinges on the ability to preserve and retrieve the non-classicality of the input quantum state, thus requiring low-noise operation for any quantum applications. Our quantum memory system of choice operates using a Raman transition in the $\Lambda$ energy level system of warm caesium vapour to store an input signal. Applying a strong control field, together with the input signal creates a 2-photon transition, storing the signal as a coherence between long-lived spin states, across the entire ensemble [2]. The Raman memory combines high-bandwidth storage, room-temperature operation, mode-selectivity, and moderate storage times. This memory offers large time-bandwidth products in excess of 1000 making it a very promising candidate for synchronisation of probabilistic operations.
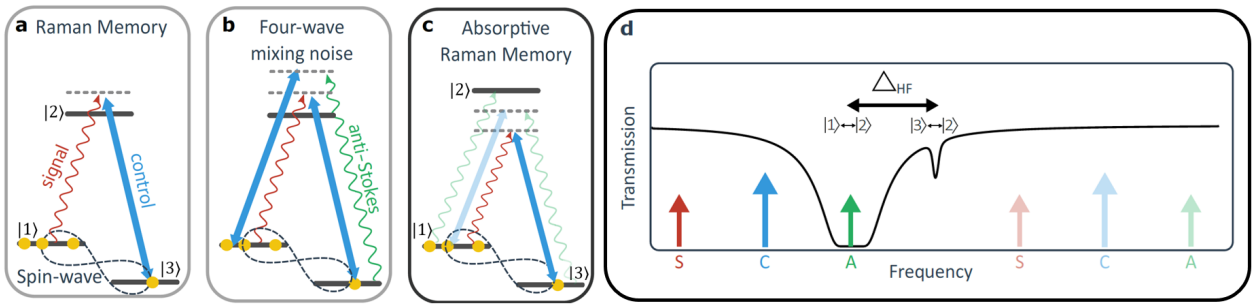

Fig. 1. a) Energy level diagram showing the fields involved in the Raman memory, the input signal is stored as an atomic coherence or spin-wave across the ensemble. b) The fields involved in the four-wave mixing noise process whereby spurious signal is produced via anti-Stokes scattering. c) The field arrangement in the absorptive noise suppression scheme where the signal and control are chosen such that the anti-Stokes is resonant with the atomic transition. d) Transmission spectrum of warm caesium vapour in a nitrogen buffer gas. The arrows show the frequency of the signal, control and anti-Stokes fields for the absorptive (left) and standard (right) Raman protocols. The anti-Stokes field is on resonance with the atomic transition in the absorptive scheme.

Of utmost importance to any memory protocol is the requirement of noise-free operation. Previous implementations of the Raman memory have suffered from four-wave mixing noise (Figure 1b) where the control couples to the populated state to produce a noise photon, and corresponding noise spin-wave by anti-Stokes scattering. This noise contaminates the retrieved fields with thermal noise, destroying the non-classical statistics of stored quantum states. This has been identified as the key limiting factor in reaching quantum level operation [3].

In the past, the inclusion of a cavity around the memory [4] (which is limited by intra-cavity loss), or a weak two-photon absorption process to another isotope [5], have been used to achieve noise-suppression. Here, we arrange the Raman memory interaction such that the anti-Stokes field is resonant with the populated transition 
$(|1\rangle \rightarrow|3\rangle$ in Figure 1d) of the same caesium isotope, thereby undergoing strong absorption and dispersion, and thus suppressing the noise.

To test the effect of this suppression method we conduct storage of weak coherent states under the two schemes by looking at how the noise level scales with the memory lifetime (Figure 2 left). For the standard Raman memory, the noise in the input time-bin is constant, but the noise in the retrieval time bin decreases with storage time.This indicates that the noise process involves an atomic coherence which decays over a similar timescale as the memory efficiency - consistent with four-wave mixing noise. For the absorptive memory the noise is independent of the storage time, indicating that we have suppressed four-wave mixing and and observe a constant background from residual technical noise such as spontaneous scattering due to imperfect optical pumping and fluorescence. These would generate the same amount of noise photons on every application of the control pulse.

To test the quantumness of the memory, we measure the second order autocorrelation function of the retrieved light as a function of coherent state amplitude. By developing a model for the output $g^{(2)}$ we are able to predict the statistics for the retrieved state, given a single photon input. We are able to show that this method could retrieve a non-classical state from a single-photon with this noise-suppression scheme, providing the heralding efficiency was greater than $27 \%$.

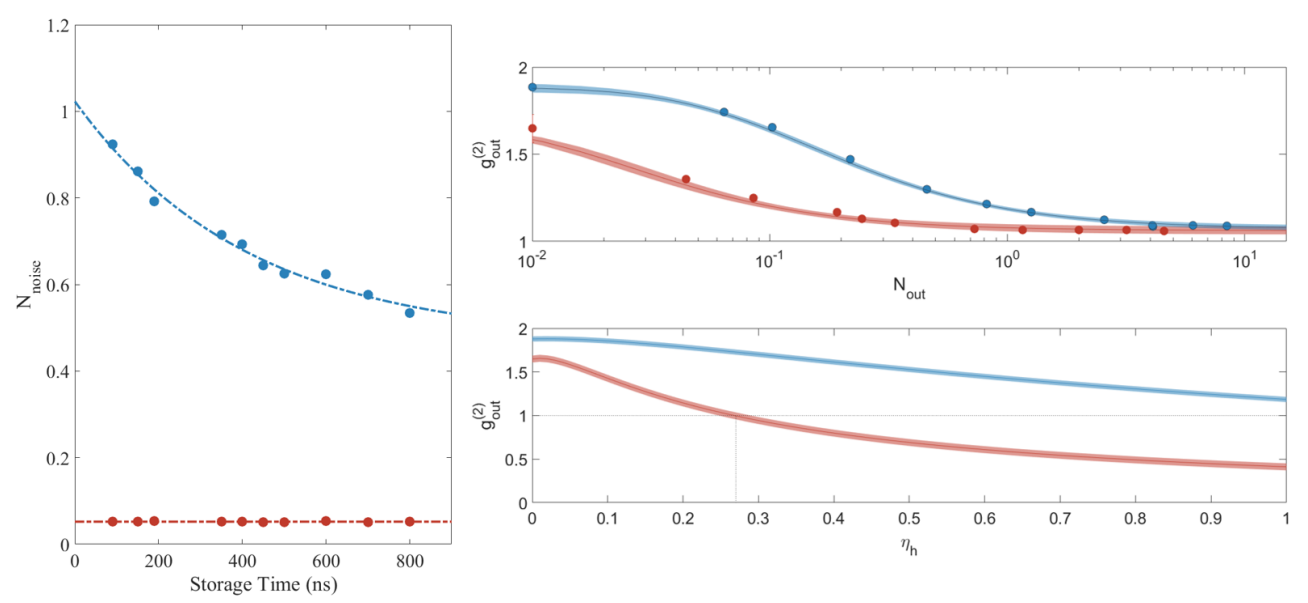

Fig. 2. Left: The measured noise photons per pulse, $N_{\text {noise }}$, retrieved from the memory as a function of storage time for the absorptive (red) and standard (blue) Raman memory. Right (Upper): The measured $g^{(2)}$ of the retrieved state from the memory as a function of the retrieved photon number, $N_{\text {out }}$ for the absorptive and standard Raman memory. The solid lines are the fit to data using the model we have developed, and the shaded regions indicate the $95 \%$ confidence intervals on the fit. (Lower): The predicted $g_{\text {out }}^{(2)}$ for a single photon input with $g_{\text {in }}^{(2)}=0.02$ as a function of the input photon number or heralding efficiency, $\eta_{h}$, using the parameters from the fit above.

(Note: error bars are within marker size)

With the potential elimination of this noise pathway, the Raman memory is a technically simple broadband and single mode memory capable of manipulating and storing an arbitrary and user-chosen quantum state in the temporal mode basis. This has many future applications including the re-shaping of the temporal wavepacket from solid state systems, such as atoms and quantum dots, for more efficient interfacing in hybrid quantum architectures. Therefore, with further engineering, the Raman memory is a promising candidate for room-temperature temporal-mode manipulation and multiplexed photon generation in future quantum networks.

\section{References}

1. J Nunn, NK Langford, WS Kolthammer, TFM Champion, MR Sprague, PS Michelberger, X-M Jin, DG England, and IA Walmsley. Phys. Rev. Lett., 110(13):133601, 2013.

2. J Nunn, IA Walmsley, MG Raymer, K Surmacz, FC Waldermann, Z Wang, and D Jaksch. Phys. Rev. A, 75(1):011401, 2007.

3. PS Michelberger, TFM Champion, MR Sprague, KT Kaczmarek, M Barbieri, XM Jin, DG England, WS Kolthammer, DJ Saunders, J Nunn, et al. New Journal of Physics, 17(4):043006, 2015.

4. DJ Saunders, JHD Munns, TFM Champion, C Qiu, KT Kaczmarek, E Poem, PM Ledingham, IA Walmsley, and J Nunn. Phys. Rev. Lett., 116(9):090501, 2016.

5. G Romanov, C OBrien, and I Novikova. Journal of Modern Optics, 63(20):2048-2057, 2016. 